\title{
A Case Report on the Impact of Physiotherapy on Shoulder Function in Breast Cancer Patients Undergoing Surgery
}

\author{
Shilpa Khurana ${ }^{1}$, Namrata Rathi ${ }^{2}$, Leena Jaiswal ${ }^{3}$ \\ ${ }^{1}$ Professor, Shri K. R. Pandav College of Physiotherapy, Bhilewada, Bhandara, India. \\ ${ }^{2}$ Professor, Department of Neurosciences Physiotherapy, Shri K. R. Pandav College of Physiotherapy, \\ Bhilewada, Bhandara, India. \\ ${ }^{3}$ Assistant Professor, Smt. Radhikatai Pandav College of Physiotherapy, Nandanvan, Nagpur.
}

Corresponding Author: Namrata Rathi

DOI: https://doi.org/10.52403/ijhsr.20220318

\begin{abstract}
Early breast cancer treatment can cause shoulder dysfunction, which is a well-known and prevalent adverse effect (1). In individuals treated surgically for breast cancer, physiotherapy was found to enhance shoulder function considerably (1). Breast cancer is the most common type of cancer in women and the leading cause of death and morbidity (2). Every year, 1.67 million new instances of breast cancer are identified worldwide, with 458,000 fatalities (2). Although 89 percent of breast cancer survivors live for at least five years after treatment, side symptoms can continue for months or even years(2). The most common upper-limb side effects are pain and joint dysfunction, with prevalence rates ranging from $12 \%$ to $51 \%$ for pain and 1.5 percent to $50 \%$ for joint dysfunction. Surgery is the most common treatment for primary breast cancer. Shoulder exercises are commonly advised to reduce mobility and strength loss as well as prevent lymphedema. Several clinical services have been developed to help with shoulder range of motion rehabilitation and secondary lymphedema prevention(3). The goal of this study was to see how additional postoperative physiotherapy affected shoulder function after the initial postoperative healing period, especially when given for a longer period. Patients who have had a mastectomy are always at risk of getting shoulder pain and adhesive capsulitis, and they must take precautions (3).
\end{abstract}

Keywords: Modified radical mastectomy, shoulder pain, breast cancer, lymph nodes, physiotherapy rehabilitation.

\section{INTRODUCTION}

A common adverse effect of early breast cancer treatment is a loss of shoulder function (1). Physiotherapy is critical in proving to patients that their shoulder can be used during the first week after surgery (1). In women over the age of 45 , it is the leading cause of death (2). Clinical management requires a multidisciplinary approach involving the skills of various medical practitioners (oncologist, surgeon, cosmetic surgeon, physiatrist, nurse, physiotherapist, radiotherapy, and psycho oncologist) to ensure an effective therapeutic outcome in terms of survival and quality of life (QOL) (2). Breast cancer surgery, particularly mastectomy, limits arm/shoulder mobility, leading to pain and fibrosis in the arms and shoulders (2). Females who have had a mastectomy had a slightly increased prevalence of shoulder morbidity, according to research (17\%) (2). Breast cancer (BC) is the primary cause of cancer death in women aged 20 to 59 (3). More than $85 \%$ of women diagnosed with $\mathrm{BC}$ live for at least 5 years, a figure that has 
increased dramatically from the $75 \%$ reported 30 years ago (3). The increase in survival rates can be linked to two factors: greater early $\mathrm{BC}$ detection rates and improved adjuvant therapy. According to the research, improving multimodal $\mathrm{BC}$ treatment predicts a higher chance of survival (3). Arm morbidity is common following first breast cancer treatment upper limb edema, soreness, impaired shoulder mobility, and other deficits have been thoroughly documented in the literature sensory as well as motor deficits (4). Breast and axillary surgery, as well as radiotherapy, cause scar tissue formation, wound formation, fibrosis, and shortening of soft tissues, such as the pectoral muscle, during the acute treatment phase of cancer(5) . The number of breast cancer survivors has risen dramatically as a result of earlier detection, better treatment, and a variety of multimodal rehabilitation options (5). However, increased breast cancer survival rates come with a slew of negative effects from the disease and its treatment. To enhance survivors' overall functioning, comprehensive rehabilitation methods are required to address the numerous long-term impacts of this therapy, including lesserknown cognitive deficiencies (4). The goal of surgery is to completely remove breast tumors, either by mastectomy or lumpectomy, and to check the health of the axillary lymph node, either by SLNB or axillary lymph node dissection (ALND) (5). Pain and physical limits are the most upsetting concerns in post-surgery rehabilitation, which is typically focused on the more obvious side effects (5). In the majority of cases, disability and shoulder pain in mastectomy patients can be reduced or even eradicated, however, most cases go unreported owing to a lack of understanding, putting the patient's psychosocial life in jeopardy (2). The loss of a breast produces soft tissue asymmetry and mass distribution through the chest wall, affecting upper-limb movements and resulting in trunk or shoulder discomfort (2). As a result, post-mastectomy patients' scapular and shoulder kinematics are frequently altered, causing them to struggle with daily activities (2). The importance of early intervention and the function of physiotherapy in the treatment of such disorders are discussed in this article.

\section{PATIENT INFORMATION}

A patient 34 years old female housewife who lives in Nashik. Hand dominance right hand. She was apparently alright 6 months back. Swelling in her left breast was a problem for her. As a result, she went to a private hospital. There she has done a mammography of the breast and she detected a lump in her left breast. Further investigations were done. She had a modified radical mastectomy with dissection of the axillary lymph nodes on October 8, 2021. Following surgery, the patient complained of pain in her anterior chest wall and limited range of motion in her left shoulder. For one month after surgery, the patient maintained a guarded posture and did the limited movement of her left upper extremity due to discomfort and anxiety of reopening the incision site. As a result, the range of motion of the right shoulder was restricted, and the anterior chest wall musculature developed fibrosis. A lack of range of motion impeded the patient's ability to perform ADLs such as reaching overhead, sleeping, completing household activities, washing her hair, cooking, and getting dressed (ROM). The patient finally sought medical care when the agony and loss of motion became unbearable. Following surgery, intensive rehabilitation is started to improve physical activity. A physiotherapy appointment was scheduled for the surgery patient.

CLINICAL FINDINGS: With both shoulders at the same level, the patient was evaluated in the supine posture. On physical examination, the patient's vital signs were afebrile, with a pulse rate of 81 beats per minute, a breathing rate of 22 breaths per minute, and a blood pressure of 120/80 $\mathrm{mm} / \mathrm{hg}$. During the general assessment, the 
cardiovascular and respiratory systems were found to be normal. Tissues around the left axilla and the left anterior chest wall were discovered to be hypermobile and fibrosis during the integumentary examination. There was no sign of infection at the incision site, which was blue-purple. The pain was measured using the Numerical Pain Rating Scale (NPRS), with the patient scoring a 5/10 at rest and a 7/10 when the affected shoulder was moved beyond 90 degrees. Tenderness over the incision site was grade 3, which means that palpation was tender, and the patient winced and retracted the limb.

The range of motion was measured using a goniometer, which included both active and passive ranges of motion. There is a limitation in left shoulder ROM. There were normal ranges in all directions for the right shoulder.

\begin{tabular}{|l|l|l|}
\hline $\begin{array}{l}\text { Shoulder } \\
\text { movements }\end{array}$ & $\begin{array}{l}\text { Left upper limb (Active } \\
\text { ROM) }\end{array}$ & $\begin{array}{l}\text { (Passive } \\
\text { ROM) }\end{array}$ \\
\hline Flexion & $80^{0}$ & $90^{0}$ \\
\hline Abduction & $75^{0}$ & $80^{0}$ \\
\hline Internal Rotation & $40^{0}$ & $45^{0}$ \\
\hline External Rotation & $44^{0}$ & $48^{0}$ \\
\hline
\end{tabular}

Manual Muscle Testing (MMT) was done to measure muscles strength of left shoulder muscles. Reduced strength of left upper limb muscles was seen.

\begin{tabular}{|l|l|}
\hline Shoulder MMT & Left upper limb \\
\hline Flexion & $3 / 5$ \\
\hline Extension & $3 / 5$ \\
\hline Abduction & $3 / 5$ \\
\hline Internal rotation & $3 / 5$ \\
\hline External rotation & $3 / 5$ \\
\hline
\end{tabular}

Girth measurement was done on both the upper limb to assess any swelling as lymph nodes of the left side was removed. No significant difference was noticed.

The intervention was designed to increase shoulder mobility and strength, scar tissue mobilization, and an overall increase in functional use of the affected arm.

\section{PHYSIOTHERAPY INTERVENTION:}

The patient received routine treatment for 15 days from a licensed physiotherapist.
The short-term goal was to educate the patient on the signs and symptoms of the illness, as well as preventative measures, a home exercise routine, shoulder ROM improvement, and pain relief. Long-term objectives included maintaining shoulder range of motion in all directions, allowing the patient to utilize the limb functionally, mobilizing scar tissue, and improving body posture. Isometric hand and forearm training began on the second postoperative day. The exercises included active flexion, abduction, active assistive, and internal and external rotation ROM movements of the shoulder joint on the third and fourth days. Passive stretching activities were performed throughout the next three days. Patients in the treatment community completed 15 physiotherapy sessions after the drain was removed, which included wall climbing, pendulum, and overhead lifting pulley, horizontal abduction, wall-wall, dorsal strengthening, position, and levator-scapula streaming activities. In this case, the patients completed the workouts at home after eight weeks. Muscles such as the rotator cuff, pectoralis muscles, rhomboids, trapezius, biceps, and serratus anterior should all be targeted. Exercises can be started with elastic bands. A 10-15 minute warm-up was followed by 20-30 minutes of prescribed activity and physical treatment, followed by a 10-minute cool-down. To improve tissue pliability, scar tissue mobilization was employed in manual treatment. A deep friction massage was conducted after 5-7 minutes of ultrasonography. As a result, the range of motion in the shoulders was enhanced. The next step was to mobilize the glenohumeral joint started with grade 1-2 mobilization and working my way up to grade 3-4 mobilization, which was successful in dissolving adhesions. Hydrocollator packs were next placed to the shoulder region for 5-10 minutes, followed by stretching of the shoulder girdle and pectoral muscles. Manual stretching was first used to teach the patient how to stretch on their own, with each stretch lasting 20 seconds and being repeated 5-7 times. 
Finally, strengthening exercises were conducted, first with help and subsequently without gravity once strength had been established. Finally, modest aid was used to operate against gravity with body weight. After completing the maximal range of motion, resistance training commenced. The goal of treatment was to improve and restore tissue mobility while also minimizing soft tissue glide constraints.

\section{DISCUSSION}

Shoulder joint ROM restriction, functional capacity decline, and lymphedema are the most prevalent postoperative consequences of MRM. Postoperative exercises that keep the arm moving are thought to enhance the quantity of lymphoid fluid produced following surgery. Seroma production can be caused by too much lymphoid fluid, and insufficient seroma drainage can result in infection, pain, and discomfort, as well as lengthier hospital admissions. (6) Patients with breast cancer should always be referred to a physical medicine and rehabilitation specialist, according to clinicians. Once a patient has been diagnosed with cancer, it is critical to eradicate and prevent the disease from returning (5). Strengthening, stretching, soft tissue mobilization, manipulation, and the use of various electrical modalities should be used to alleviate discomfort, enhance strength, and improve range of motion after dysfunction is recognized. A multidisciplinary approach is required to achieve a positive outcome and enhance the quality of life.

\section{CONCLUSION}

According to the findings, physiotherapy is more beneficial in reducing discomfort and improving shoulder range of motion after a modified radical mastectomy. Physiotherapy reduces discomfort and improves shoulder function and quality of life following axillary dissection after breast cancer.

\section{Author's Contribution}

Each author contributed the most to the concept, assessment and evaluation, data collecting, analysis, and interpretation of the results.

\section{Informed Consent}

The patient's consent was obtained to prepare the case report.

\section{Funding Support}

There are no financial conflicts of interest that need to be revealed.

\section{Conflict of Interest}

The authors have no conflicts of interest to disclose.

\section{Acknowledgement: None}

\section{REFERENCES}

1. Lauridsen MC, Christiansen P, Hessov I. The effect of physiotherapy on shoulder function in patients surgically treated for breast cancer: A randomized study. Acta Oncol. 2005 Jan 1;44(5):449-57.

2. Naqvi WM. The Efficacy Of Physiotherapy Recovery After A Modified Radical Mastectomy. J Med Pharm Allied Sci. 2021 Jul 15;2857-60.

3. Gomide LB, Matheus JPC, Candido dos Reis FJ. Morbidity after breast cancer treatment and physiotherapeutic performance. Int J Clin Pract. 2007; 61(6): 972-82.

4. Fourie WJ, Robb KA. Physiotherapy management of axillary web syndrome following breast cancer treatment: Discussing the use of soft tissue techniques. Physiotherapy. 2009 Dec 1;95(4):314-20.

5. Loh SY, Musa AN. Methods to improve rehabilitation of patients following breast cancer surgery: a review of systematic reviews. Breast Cancer Targets Ther. 2015;7:81.

6. Shamley DR, Barker K, Simonite V, Beardshaw A. Delayed versus immediate exercises following surgery for breast cancer: a systematic review. 
Shilpa Khurana et.al. A case report on the impact of physiotherapy on shoulder function in breast cancer patients undergoing surgery.

Breast Cancer Res Treat. 2005 Apr 1;90(3):263-71.

7. Kilgour RD, Jones DH, Keyserlingk JR. How to cite this article: Khurana S, Rathi N, Effectiveness of a self-administered, home-based exercise rehabilitation program for women following a modified radical mastectomy and axillary node dissection: a preliminary study. Breast Cancer Res Treat. 2008 Jaiswal L. A case report on the impact of physiotherapy on shoulder function in breast cancer patients undergoing surgery. Int $J$ Health Sci Res. 2022; 12(3):133-137. DOI: https://doi.org/10.52403/ijhsr.20220318 May 1;109(2):285-95. 